Errno::ENOENT: No such file or directory @ rb_sysopen - main.scss

\title{
Encyclopedia
}

\section{Economic Centrality}

Created by: Nuno Crespo

Version received: 12 November 2019

check for updates

A basic index of economic centrality is proposed. This measure makes clear the influence of internal and external dimensions as well as economic and geographical impacts. Some critical policy actions that can be taken in order to improve the centrality level of a country are discussed.

\section{Table of Content [Hide]}

The importance of centrality - i.e., closeness to markets - is widely recognized as an important advantage for the countries. This is a key advantage of countries such as Belgium or Netherlands, which exhibit the most favorable positions in the empirical analyses of economic centrality.

How can we measure this concept? Several proposals were advanced since the pioneering contributions by Keeble et al. ${ }^{[1][2]}$. Here, we discuss the measure introduced by Crespo, Fontoura, and Simoes ${ }^{3]}$.

The index of economic centrality can be expressed as follows:

$C_{i}=\frac{l_{i}}{\delta_{i i}}+\sum_{h} \frac{l_{h}}{\delta_{i h}}, i \neq h$

$\delta_{i i}$ represents the internal distance (which is associated with the area of the country), $\delta_{i h}$ is the distance between $i$ and $h, l_{i}$ is the share of country $i$ in sum of the values of the GDP of the countries under consideration. The same definition applies to $l_{h}$.

Equation (1) makes clear that the level of centrality exhibited by countryi depends on four dimensions, covering geographical and economic aspects both at internal and external levels. However, it does not allow to identify in which form each component contributes to the global score of the country.

In order to take a step forward in this analysis, a decomposition approach can be applied. In what follows, we discuss a method that allows to decompose the global index into four parcels with specific economic interpretation associated to the several dimensions that are incorporated in the measure. This is obtained as follows:

$C_{i}=\underbrace{\frac{\frac{1}{N}}{\delta_{i i}}}_{A}+\underbrace{\frac{\left(l_{i}-\frac{1}{N}\right)}{\overline{\delta_{i i}}} \frac{\overline{\delta_{i i}}}{\delta_{i i}}}_{B}+\underbrace{\sum_{h} \frac{\frac{1}{N}}{\delta_{i h}}}_{C}+\underbrace{\sum_{h} \frac{\left(l_{h}-\frac{1}{N}\right)}{\delta_{i h}}}_{D}$

The internal geographical component (A) is captured by the first term in (2). This assumes an equal distribution of economic activity. Thus, the values obtained by each country only depend (negatively) on its geographical dimension, evaluated through its area, as commonly done in this type of measures. If the same portion of economic activity is 
located in a smaller country then we will say that this country is more central than another one with a larger dimension, where the economic activity is more dispersed.

In turn, the internal economic component is measured through (B), being (B1) - the first term - a pure internal economic component and (B2) - the second term - a geographical adjustment factor. (B1) assumes a positive value when an above-average share of economic activity is located in that country, indicating that its centrality level benefits from a favorable position in economic terms. A negative value occurs when the country captures a below-average fraction of economic activity. Given that the internal distance is fixed at its average level, the differences between countries are fully attributable to this economic effect. Regarding (B2), it registers a value above 1 when the country is (geographically) smaller than the average and below 1 in the opposite case. The global effect $(B)$ captures the internal economic component adjusted by the dimension of the country.

The external geographical component is at the heart of the centrality concept, being evaluated, in this method, by (C). It assumes, once again, as in (A), the equal distribution of economic activity in space and analyzes how far country $i$ is from the remaining countries. More remote countries suffer from what is sometimes called as the "tyranny of distance". This expression is particularly adequate since it reflects quite well the fact that a negative position in this aspect is extremely difficult to minimize and impossible to overcome in its full extension.

Finally, we should also consider the distribution of economic activity among the other countries. However, it is important to note that, by opposition to the preceding components, we cannot isolate a pure external economic component. The reason for that is straightforward. Obviously, the share of economic activity located outside $i$ is (1- This does not give us any new insight. What really matters is the spatial distribution of that part of the total economic activity and, more specifically, its closeness to $i$. Therefore, (D) is influenced both by economic and geographical aspects, assuming a positive value when economic advantages are obtained by countries closer to $i$. Its minimum value is reached, for $i$, when the economic activity is fully concentrated in the farthest country.

One of the most important insights allowed by this decomposition methodology is that it offers guidance for policy interventions aiming to improve the global level of centrality. Effectively, distinct policy measures can be recommended depending on the main weaknesses detected. Let us consider each specific component. The improvement on the internal geographical component (A) can be obtained through better infrastructures, such as roads, which allow a reduction on transport costs and times. In turn, if a country shows a low score on the internal economic component (B), interventions should be devoted to the attraction of more economic activity to the country, for instance through favorable conditions to FDI. In turn, rapid access to external countries is vital to improve centrality through component (C). The creation and/or improvement of infrastructures that connect the country to foreign countries are adequate interventions to improve centrality. Component (D) is the only one that is out of control of national authorities. It depends on the distribution of economic activity across the remaining countries, an aspect that national policymakers do not influence in a direct way. Nevertheless, an indirect aspect may contribute to improve this component, namely the formation of regional integration blocks, with the elimination (or, at least, reduction) of trade barriers between the members of the block. This may attract more economic activity for the whole block, which is usually composed by adjacent countries.

\section{References}

1. Keeble, D., Offord, J. and Walker, S. 1988, "Peripheral regions in a community of twelve Member States", Report for the European Commission, Brussels.

2. Keeble, D., Offord, J. and Walker, S. 1988, "Peripheral regions in a community of twelve Member States", Report for the European Commission, Brussels.

3. Crespo, N., M. Fontoura and N. Simoes (2014), "Economic Centrality: How Much is Economics and How Much is Geography?”, MPRA Working Paper No. 58028.

\section{Keywords}

economic centrality; geography; index

(C) 2019 by the author(s). Distribute under aCreative Commans CC BY license 